\title{
Convergence Theorem for Equilibrium and Variational Inequality Problems and a Family of Infinitely Nonexpansive Mappings in Hilbert Space
}

\author{
Zhou Yinying, Cao Jiantao, and Wang Yali \\ Department of Mathematics and Information Sciences, Langfang Teachers College, Langfang, Hebei 065000, China \\ Correspondence should be addressed to Zhou Yinying; zhouyinying_hbu@163.com
}

Received 1 January 2014; Accepted 28 February 2014; Published 7 April 2014

Academic Editor: Li Wei

Copyright (C) 2014 Zhou Yinying et al. This is an open access article distributed under the Creative Commons Attribution License, which permits unrestricted use, distribution, and reproduction in any medium, provided the original work is properly cited.

We introduce a hybrid iterative scheme for finding a common element of the set of common fixed points for a family of infinitely nonexpansive mappings, the set of solutions of the varitional inequality problem and the equilibrium problem in Hilbert space. Under suitable conditions, some strong convergence theorems are obtained. Our results improve and extend the corresponding results in (Chang et al. (2009), Min and Chang (2012), Plubtieng and Punpaeng (2007), S. Takahashi and W. Takahashi (2007), Tada and Takahashi (2007), Gang and Changsong (2009), Ying (2013), Y. Yao and J. C. Yao (2007), and Yong-Cho and Kang (2012)).

\section{Introduction}

Let $H$ be a real Hilbert space, whose inner product and norm are denoted by $\langle\cdot, \cdot\rangle$ and $\|\cdot\|$, respectively. Let $C$ be a nonempty closed convex subset of $H$ and $P_{C}$ the metric projection of $H$ onto $C$. Let $\phi: C \times C \rightarrow R$ be a bifunction. We consider the equilibrium problem EP which is to find $z \in C$ such that

$$
\phi(z, y) \geq 0, \quad \forall y \in C .
$$

Let $\operatorname{EP}(\phi)$ be the set of solutions. Some methods have been proposed to solve the equilibrium problem.

A mapping $A$ is said to be $\alpha$-inverse strongly monotone if there exists a real number $\alpha>0$ such that $\langle A x-A y, x-y\rangle \geq$ $\alpha\|A x-A y\|^{2}$, for all $x, y \in C$.

The classical variational inequality problem is to find an element $u \in C$ such that

$$
\langle A u, v-u\rangle \geq 0, \quad \forall v \in C .
$$

The solution set of inequality (2) is denoted by $\operatorname{VI}(C, A)$. For given elements $z \in H$ and $u \in C$, we have the following inequality:

$$
\langle u-z, v-u\rangle \geq 0, \quad \forall v \in C,
$$

if and only if $u=P_{C} z$. It is known that the projection operator $P_{C}$ is nonexpansive. One can see that the variational inequality problem (2) is equivalent to a fixed point problem. Since an element $u \in C$ is the solution of variational inequality (2) if and only if $u \in C$ is a fixed point of the mapping $P_{C}(I-\lambda \mathrm{A})$. Recently, many researchers studied various iterative algorithms for finding an element of $\operatorname{VI}(C, A) \cap F(S)$. Takahashi and Toyoda [1] introduced the following iterative scheme:

$$
x_{n+1}=\alpha_{n} x_{n}+\left(1-\alpha_{n}\right) S P_{C}\left(I-\lambda_{n} B\right) x_{n}, \quad \forall n \geq 0 .
$$

They proved that the sequence $\left\{x_{n}\right\}$ converges weakly to a point $q \in \mathrm{VI}(C, B) \cap F(S)$. Y. Yao and J. C. Yao [2] introduced the following iterative scheme:

$$
\begin{gathered}
x_{1}=u \in C, \\
y_{n}=P_{C}\left(I-\lambda_{n} A\right) x_{n}, \\
x_{n+1}=\alpha_{n} u+\beta_{n} x_{n}+\gamma_{n} S P_{C}\left(I-\lambda_{n} A\right) y_{n} .
\end{gathered}
$$


Chang et al. [3] introduced the following iterative scheme:

$$
\begin{gathered}
\phi\left(u_{n}, y\right)+\frac{1}{r_{n}}\left\langle y-u_{n}, u_{n}-x_{n}\right\rangle, \quad \forall y \in C, \\
x_{n+1}=\alpha_{n} f\left(x_{n}\right)+\beta_{n} x_{n}+\gamma_{n} W_{n} k_{n}, \\
k_{n}=P_{C}\left(I-\lambda_{n} B\right) y_{n}, \\
y_{n}=P_{C}\left(I-\lambda_{n} B\right) u_{n},
\end{gathered}
$$

and obtained some strong convergence theorems.

In this paper, we will introduce a new hybrid iterative scheme for finding a common element of the set of common fixed points for a family of infinitely nonexpansive mappings, the set of solutions of the variational inequality problem, and the equilibrium problem. Further, we obtain some strong convergence theorems and extend the results in [2-10].

\section{Preliminaries}

Let $x_{n} \rightarrow x$ and $x_{n} \rightarrow x$ be the weak convergence and strong convergence of the sequence $\left\{x_{n}\right\}$ in $H$. Let $C$ be a nonempty closed convex subset of a Hilbert space $H$. Let $\left\{S_{n}\right\}_{n=1}^{\infty}: C \rightarrow C$ be a family of infinitely nonexpansive mappings and let $\left\{\lambda_{n}\right\}_{n=1}^{\infty}$ be a sequence of positive numbers in $[0,1]$. For $n \geq 1$, we define a mapping $W_{n}: C \rightarrow C$ as follows:

$$
\begin{aligned}
& U_{n, n+1}=I \\
& U_{n, n}=\lambda_{n} S_{n} U_{n, n+1}+\left(1-\lambda_{n}\right) I \\
& U_{n, n-1}=\lambda_{n-1} S_{n-1} U_{n, n}+\left(1-\lambda_{n-1}\right) I \\
& \vdots \\
& U_{n, k}=\lambda_{k} S_{k} U_{n, k+1}+\left(1-\lambda_{k}\right) I \\
& U_{n, k-1}=\lambda_{k-1} S_{k-1} U_{n, k}+\left(1-\lambda_{k-1}\right) I \\
& \vdots \\
& U_{n, 2}=\lambda_{2} S_{2} U_{n, 3}+\left(1-\lambda_{2}\right) I \\
& W_{n}=U_{n, 1}=\lambda_{1} S_{1} U_{n, 2}+\left(1-\lambda_{1}\right) I .
\end{aligned}
$$

$W_{n}$ is the $W$-mapping of $C$ into itself which is generated by $S_{n}, S_{n-1}, \ldots, S_{1}$ and $\lambda_{n}, \lambda_{n-1}, \ldots, \lambda_{1}$.

In order to prove our main results, the following Lemmas are needed.

Lemma 1 (see [11]). Let $C$ be a nonempty closed convex subset of a Banach space E, let $\left\{S_{n}\right\}_{n=1}^{\infty}: C \rightarrow C$ be a family of infinitely nonexpansive mappings, such that $\bigcap_{n=1}^{\infty} F\left(S_{n}\right) \neq \emptyset$, and let $\left\{\lambda_{n}\right\}_{n=1}^{\infty}$ be a sequence of positive numbers in $[0, b]$ for some $b \in(0,1)$. For any $n \geq 1$, let $\left\{W_{n}\right\}$ be the $W$-mapping of $C$ into itself generated by $S_{n}, S_{n-1}, \ldots, S_{1}$ and $\lambda_{n}, \lambda_{n-1}, \ldots, \lambda_{1}$. Then $W_{n}$ is asymptotically regular and nonexpansive. Further, if $E$ is strictly convex, then $F\left(W_{n}\right)=\bigcap_{i=1}^{n} F\left(S_{i}\right)$.
Lemma 2 (see [4]). Let $C$ be a nonempty closed convex subset of a strictly convex Banach space E. Let $\left\{S_{n}\right\}_{n=1}^{\infty}$ : $C \rightarrow C$ be a family of infinitely nonexpansive mappings, such that $\bigcap_{n=1}^{\infty} F\left(S_{n}\right) \neq \emptyset$, and let $\left\{\lambda_{n}\right\}_{n=1}^{\infty}$ be a sequence of positive numbers in $[0, b]$ for some $b \in(0,1)$. Then for every $x \in C$ and $k \geq 1 \lim _{k \rightarrow \infty} U_{n, k} x$ exists.

Using Lemma 2, we can define a mapping $W: C \rightarrow C$ as follows:

$$
W x=\lim _{n \rightarrow \infty} W_{n} x=\lim _{n \rightarrow \infty} U_{n, 1} x, \quad \forall x \in C .
$$

Such a $W$ is called the $W$-mapping generated by the sequence $\left\{S_{n}\right\}_{n=1}^{\infty}$ and $\left\{\lambda_{n}\right\}_{n=1}^{\infty}$. Throughout this paper, we always assume that $\left\{\lambda_{n}\right\}_{n=1}^{\infty}$ is a sequence of positive numbers in $[0, b]$ for an element $b \in(0,1)$.

Lemma 3 (see [4]). Let $C$ be a nonempty closed convex subset of a strictly convex Banach space E. Let $\left\{S_{n}\right\}_{n=1}^{\infty}$ : $C \rightarrow C$ be a family of infinitely nonexpansive mappings such that $\bigcap_{n=1}^{\infty} F\left(S_{n}\right) \neq \emptyset$ and let $\left\{\lambda_{n}\right\}_{n=1}^{\infty}$ be a sequence of positive numbers in $[0, b]$ for some $b \in(0,1)$. Then, $W$ is $a$ nonexpansive mapping and $F(W)=\bigcap_{n=1}^{\infty} F\left(S_{n}\right)$.

Lemma 4 (see [4]). Let $C$ be a nonempty closed convex subset of a Hilbert space $H$, let $\left\{S_{n}\right\}_{n=1}^{\infty}: C \rightarrow C$ be a family of infinitely nonexpansive mappings, such that $\bigcap_{n=1}^{\infty} F\left(S_{n}\right) \neq \emptyset$, and let $\left\{\lambda_{n}\right\}_{n=1}^{\infty}$ be a sequence of positive numbers in $[0, b]$ for some $b \in(0,1)$. If $K$ is any bounded subset of $C$, then $\lim _{n \rightarrow \infty} \sup _{x \in K}\left\|W x-W_{n} x\right\|=0$.

Lemma 5 (see [10]). Let $\left\{x_{n}\right\}$ and $\left\{z_{n}\right\}$ be bounded sequences in a Banach space $E$ and let $\left\{\beta_{n}\right\}$ be a sequence in $[0,1]$ with $0<\liminf _{n \rightarrow \infty} \beta_{n} \leq \limsup _{n \rightarrow \infty} \beta_{n}<1$. Suppose that $x_{n+1}=$ $\left(1-\beta_{n}\right) z_{n}+\beta_{n} x_{n}$ for all integers $n \geq 1$ and $\lim \sup _{n \rightarrow \infty}\left(\| z_{n+1}-\right.$ $\left.z_{n}\|-\| x_{n+1}-x_{n} \|\right) \leq 0$. Then, $\lim _{n \rightarrow \infty}\left\|z_{n}-x_{n}\right\|=0$.

Lemma 6 (see [10]). Assume that $a_{n}$ is a sequence of nonnegative real numbers, such that

$$
a_{n+1} \leq\left(1-\gamma_{n}\right) a_{n}+\delta_{n}, \quad \forall n \geq n_{0}
$$

where $n_{0}$ is some nonnegative integer, $\gamma_{n} \in(0,1)$, and $\delta_{n}$ are sequences satisfying

(1) $\sum_{n=1}^{\infty} \gamma_{n}=\infty$;

(2) $\lim \sup _{n \rightarrow \infty}\left(\delta_{n} / \gamma_{n}\right) \leq 0$ or $\sum_{n=1}^{\infty}\left|\delta_{n}\right|=\infty$; then, $\lim _{n \rightarrow \infty} a_{n}=0$.

Lemma 7 (see [10]). Let $C$ be a nonempty closed convex subset of a Hilbert space $H$. Let $\left\{T_{m}: 1 \leq m \leq r\right\}$ be a sequence of nonexpansive mappings on $C$. Suppose that $\bigcap_{m=1}^{r} F\left(T_{m}\right)$ is nonempty. Let $\left\{\lambda_{m}\right\}$ be a sequence of positive numbers with $\sum_{m=1}^{r} \lambda_{m}=1$. Then, a mapping $S$ on $C$ defined by $S x=$ $\sum_{m=1}^{r=1} \lambda_{m} T_{m} x$ for all $x \in C$ is well defined and nonexpansive and $F(S)=\bigcap_{m=1}^{r} F\left(T_{m}\right)$ holds.

For solving the equilibrium problem for bifunction $F$ : $C \times C \rightarrow R$, assume that $F$ satisfies the following conditions:

$\left(A_{1}\right) F(x, x)=0$ for all $x \in C$; 
$\left(A_{2}\right) F$ is monotone; that is, $F(x, y)+F(y, x) \leq 0$ for all $x, y \in C$;

$\left(A_{3}\right) \lim _{t \rightarrow 0} F(t z+(1-t) x, y) \leq F(x, y)$, for any $x, y, z \in C$;

$\left(A_{4}\right)$ for each $x \in C, y \mapsto F(x, y)$ is convex and lower semicontinuous.

If an equilibrium bifunction $F: C \times C \rightarrow R$ satisfies conditions $\left(A_{1}\right)-\left(A_{4}\right)$, then we have the following two important results.

Lemma 8 (see [4]). Let $C$ be a nonempty closed convex subset of a Hilbert space $H$ and let $F$ be an equilibrium bifunction $F: C \times C \rightarrow R$ that satisfies conditions $\left(A_{1}\right)-\left(A_{4}\right)$. Let $r>0$ and $x \in C$; then, there exists $y \in C$ such that $F(y, z)+(1 / r)\langle z-$ $y, y-x\rangle \geq 0$, for all $z \in C$.

Lemma 9 (see [4]). Let $F$ be an equilibrium bifunction $F: C \times$ $C \rightarrow R$ that satisfies conditions $\left(A_{1}\right)-\left(A_{4}\right)$. For given $r>0$ and $x \in C$, define a mapping $V_{r}: H \rightarrow C$ as follows:

$$
V_{r}(x)=\left\{y \in C: F(y, z)+\frac{1}{r}\langle z-y, y-x\rangle \geq 0, \forall z \in C\right\} .
$$

Then, the following conclusions hold:

(1) $V_{r}$ is single-valued;

(2) $V_{r}$ is firmly nonexpansive; that is, for any $x, y \in H$, $\left\|V_{r} x-V_{r} y\right\|^{2} \leq\left\langle V_{r} x-V_{r} y, x-y\right\rangle$;

(3) $F\left(V_{r}\right)=\operatorname{EP}(F)$

(4) $\mathrm{EP}(F)$ is a closed and convex set.

\section{The Main Results}

Theorem 10. Let $C$ be a nonempty closed convex subset of a real Hilbert space $H$. Let $B_{m}: C \rightarrow H$ be a $l_{m}$-inverse strongly monotone mapping for each $1 \leq m \leq r$, where $r$ is some positive integer. Let $D: C \rightarrow H$ be a $\alpha$-inverse strongly monotone mapping. Let $F$ be an equilibrium bifunction $F: C \times C \rightarrow$ $R$ that satisfies conditions $\left(A_{1}\right)-\left(A_{4}\right)$. Let $\left\{T_{n}\right\}_{n=1}^{\infty}: C \rightarrow C$ be a family of infinite $k_{n}$-strict pseudocontractive mappings with $0 \leq k_{n}<1$ and let $\left\{\lambda_{n}\right\}_{n=1}^{\infty}$ be a sequence of positive numbers in $[0, b]$ for some $b \in(0,1) .\left\{S_{n}\right\}_{n=1}^{\infty}: C \rightarrow C$ is a family of infinitely nonexpansive mappings such that $\mathscr{F}=$ $F(W) \bigcap \operatorname{VI}\left(C, B_{m}\right) \bigcap \mathrm{EP} \neq \emptyset$, where $F(W):=\bigcap_{n=1}^{\infty} F\left(S_{n}\right)$. Let $A$ be a strongly positive linear bounded operator with coefficient $\bar{\gamma}>0$ and let $f: H \rightarrow H$ be a contraction with contraction constant $h(0<h<1)$ and $0<\gamma<(\bar{\gamma} / h)$. Let $\left\{x_{n}\right\},\left\{y_{n}\right\},\left\{\rho_{n}\right\}$ be sequence generated by $x_{1} \in H$ and

$$
\begin{gathered}
F\left(y_{n}, \eta\right)+\left\langle D y_{n}, \eta-y_{n}\right\rangle+\frac{1}{r_{n}}\left\langle\eta-y_{n}, y_{n}-x_{n}\right\rangle \geq 0, \\
\forall \eta \in C, \\
\rho_{n}=\sum_{m=1}^{r} \eta_{n}^{m} P_{C}\left(I-\mu_{m} B_{m}\right) y_{n}, \\
x_{n+1}=\alpha_{n} \gamma f\left(x_{n}\right)+\beta_{n} x_{n}+\left(\left(1-\beta_{n}\right) I-\alpha_{n} A\right) W_{n} \rho_{n},
\end{gathered}
$$

where $\mu_{m} \in\left(0,2 l_{m}\right),\left\{\alpha_{n}\right\},\left\{\beta_{n}\right\} \subset[0,1]$, and $\left\{r_{n}\right\} \subset[0, \infty]$. If the following conditions are satisfied:

$\left(C_{1}\right) \lim _{n \rightarrow \infty} \alpha_{n}=0$ and $\sum_{n=1}^{\infty} \alpha_{n}=\infty$;

$\left(C_{2}\right) \lim _{n \rightarrow \infty} \eta_{n}^{m}=\eta^{m} \in(0,1)$;

$\left(C_{3}\right) \sum_{n=1}^{\infty}\left|r_{n+1}-r_{n}\right|<\infty$;

$\left(C_{4}\right) \liminf _{n \rightarrow \infty} r_{n}>0,0<\liminf _{n \rightarrow \infty} \beta_{n} \leq$ $\lim \sup _{n \rightarrow \infty} \beta_{n}<1$;

$\left(C_{5}\right) \sum_{n=1}^{\infty} \eta_{n}^{m}=1$, for all $n \geq 1$,

then $\left\{x_{n}\right\}$ converges strongly to $q \in \mathscr{F}$, where $q=P_{\mathscr{F}}(\gamma f+(I-$ A))q.

Proof. We define a bifunction $\phi: C \times C \rightarrow R$ by $\phi(z, y)=$ $F(z, y)+\langle D z, y-z\rangle$, for all $y, z \in C$, so the equilibrium problem is equivalent to the following equilibrium problem: find an element $z \in C$ such that $\phi(z, y) \geq 0$, for all $y \in C$ and (11) can be written as

$$
\begin{gathered}
\phi\left(y_{n}, \eta\right)+\frac{1}{r_{n}}\left\langle\eta-y_{n}, y_{n}-x_{n}\right\rangle \geq 0, \quad \forall \eta \in C, \\
\rho_{n}=\sum_{m=1}^{r} \eta_{n}^{m} P_{C}\left(I-\mu_{m} B_{m}\right) y_{n}, \\
x_{n+1}=\alpha_{n} \gamma f\left(x_{n}\right)+\beta_{n} x_{n}+\left(\left(1-\beta_{n}\right) I-\alpha_{n} A\right) W_{n} \rho_{n} .
\end{gathered}
$$

Step 1. First, we prove the sequences $\left\{x_{n}\right\},\left\{y_{n}\right\},\left\{\rho_{n}\right\}$ are bounded.

Let $p \in \mathscr{F}$; as $y_{n}=V_{r_{n}} x_{n}$, we have $\left\|y_{n}-p\right\|=\| V_{r_{n}} x_{n}-$ $p\|\leq\| x_{n}-p \|$. Next we show that the mapping $I-\mu_{m} B_{m}$ is nonexpansive for each $m$. Consider

$$
\begin{aligned}
\|(I- & \left.\mu_{m} B_{m}\right) x-\left(I-\mu_{m} B_{m}\right) y \|^{2} \\
= & \left\|(x-y)-\mu_{m}\left(B_{m} x-B_{m} y\right)\right\|^{2} \\
= & \|x-y\|^{2}+\mu_{m}^{2}\left\|B_{m} x-B_{m} y\right\|^{2} \\
& -2 \mu_{m}\left\langle B_{m} x-B_{m} y, x-y\right\rangle \\
\leq & \|x-y\|^{2}+\mu_{m}^{2}\left\|B_{m} x-B_{m} y\right\|^{2}-2 \mu_{m} l_{m}\left\|B_{m} x-B_{m} y\right\|^{2} \\
= & \|x-y\|^{2}-\mu_{m}\left(2 l_{m}-\mu_{m}\right)\left\|B_{m} x-B_{m} y\right\|^{2} \\
\leq & \|x-y\|^{2}, \\
\| \rho_{n}- & p\|=\| \sum_{m=1}^{r} \eta_{n}^{m} P_{C}\left(I-\mu_{m} B_{m}\right) y_{n}-p \| \\
& \leq \sum_{m=1}^{r} \eta_{n}^{m}\left\|y_{n}-p\right\| \leq\left\|x_{n}-p\right\| .
\end{aligned}
$$


Since $A$ is a strongly positive linear bounded operator, then $\|A\|=\sup \{|\langle A u, u\rangle|: u \in H,\|u\|=1\},\left\langle\left(\left(1-\beta_{n}\right) I-\right.\right.$ $\left.\left.\alpha_{n} A\right) u, u\right\rangle=1-\beta_{n}-\alpha_{n}\langle A u, u\rangle \geq 1-\beta_{n}-\alpha_{n}\|A\| \geq 0$, so

$$
\begin{aligned}
\|(1- & \left.\beta_{n}\right) I-\alpha_{n} A \| \\
& =\sup \left\{\left|\left\langle\left(\left(1-\beta_{n}\right) I-\alpha_{n} A\right) u, u\right\rangle\right|: u \in H,\|u\|=1\right\} \\
& \leq 1-\beta_{n}-\alpha_{n} \bar{\gamma},
\end{aligned}
$$$$
\left\|\left(1-\beta_{n}\right) I-\alpha_{n} A\right\|
$$$$
=\sup \left\{\left|\left\langle\left(\left(1-\beta_{n}\right) I-\alpha_{n} A\right) u, u\right\rangle\right|: u \in H,\|u\|=1\right\}
$$$$
\leq 1-\beta_{n}-\alpha_{n} \bar{\gamma}
$$$$
\left\|x_{n+1}-p\right\|
$$$$
=\left\|\alpha_{n} \gamma f\left(x_{n}\right)+\beta_{n} x_{n}+\left(\left(1-\beta_{n}\right) I-\alpha_{n} A\right) W_{n} \rho_{n}-p\right\|
$$$$
=\| \alpha_{n} \gamma\left(f\left(x_{n}\right)-f(p)\right)+\beta_{n}\left(x_{n}-p\right)
$$$$
+\left(\left(1-\beta_{n}\right) I-\alpha_{n} A\right)\left(W_{n} \rho_{n}-p\right)
$$$$
+\alpha_{n}(\gamma f(p)-A p) \|
$$$$
\leq \alpha_{n} \gamma h\left\|x_{n}-p\right\|+\beta_{n}\left\|x_{n}-p\right\|
$$$$
+\left(\left(1-\beta_{n}\right) I-\alpha_{n} A\right)\left\|x_{n}-p\right\|+\alpha_{n}\|\gamma f(p)-A p\|
$$$$
\leq \alpha_{n} \gamma h\left\|x_{n}-p\right\|+\beta_{n}\left\|x_{n}-p\right\|+\left(1-\beta_{n}\right)\left\|x_{n}-p\right\|
$$$$
-\alpha_{n} \bar{\gamma}\left\|x_{n}-p\right\|+\alpha_{n}\|\gamma f(p)-A p\|
$$$$
=\left(1-\alpha_{n}(\bar{\gamma}-\gamma h)\right)\left\|x_{n}-p\right\|+\alpha_{n}\|\gamma f(p)-A p\|
$$$$
\leq \max \left\{\left\|x_{n}-p\right\|, \frac{1}{\bar{\gamma}-\gamma h}\|\gamma f(p)-A p\|\right\}
$$$$
\vdots
$$$$
\leq \max \left\{\left\|x_{1}-p\right\|, \frac{1}{\bar{\gamma}-\gamma h}\|\gamma f(p)-A p\|\right\} .
$$

$$
\begin{aligned}
= & \frac{\alpha_{n+1}}{1-\beta_{n+1}}\left[\gamma f\left(x_{n+1}\right)-A W_{n+1} \rho_{n+1}\right] \\
& -\frac{\alpha_{n}}{1-\beta_{n}}\left[\gamma f\left(x_{n}\right)-A W_{n} \rho_{n}\right] \\
& +\left(W_{n+1} \rho_{n+1}-W_{n+1} \rho_{n}\right)+\left(W_{n+1} \rho_{n}-W_{n} \rho_{n}\right), \\
\| z_{n+1}- & z_{n}\|-\| x_{n+1}-x_{n} \| \\
\leq & \frac{\alpha_{n+1}}{1-\beta_{n+1}}\left(\left\|\gamma f\left(x_{n+1}\right)\right\|+\left\|A W_{n+1} \rho_{n+1}\right\|\right) \\
& +\frac{\alpha_{n}}{1-\beta_{n}}\left(\left\|\gamma f\left(x_{n}\right)\right\|+\left\|A W_{n} \rho_{n}\right\|\right) \\
& +\left\|\rho_{n+1}-\rho_{n}\right\|+\left\|W_{n+1} \rho_{n}-W_{n} \rho_{n}\right\|-\left\|x_{n+1}-x_{n}\right\| .
\end{aligned}
$$

Because $\rho_{n}=\sum_{m=1}^{r} \eta_{n}^{m} P_{C}\left(I-\mu_{m} B_{m}\right) y_{n}$, we have

This implies that $\left\{x_{n}\right\}$ is bounded sequence in $H$. Therefore $\left\{y_{n}\right\},\left\{\rho_{n}\right\},\left\{\gamma f\left(x_{n}\right)\right\},\left\{W_{n} \rho_{n}\right\}$ are all bounded.

Step 2. Next, we prove that $\lim _{n \rightarrow \infty}\left\|x_{n+1}-x_{n}\right\|=0$ and $\lim _{n \rightarrow \infty}\left\|\rho_{n+1}-\rho_{n}\right\|=0$.

In fact, let us define a sequence $\left\{z_{n}\right\}$ by $x_{n+1}=\left(1-\beta_{n}\right) z_{n}+$ $\beta_{n} x_{n}$, for all $n \geq 1$; then, we have

$$
\begin{aligned}
z_{n+1}- & z_{n} \\
& =\frac{x_{n+2}-\beta_{n+1} x_{n+1}}{1-\beta_{n+1}}-\frac{x_{n+1}-\beta_{n} x_{n}}{1-\beta_{n}} \\
& =\frac{\alpha_{n+1} \gamma f\left(x_{n+1}\right)+\left(\left(1-\beta_{n+1}\right) I-\alpha_{n+1} A\right) W_{n+1} \rho_{n+1}}{1-\beta_{n+1}}
\end{aligned}
$$

$$
\begin{aligned}
& \left\|\rho_{n+1}-\rho_{n}\right\| \\
& =\left\|\sum_{m=1}^{r} \eta_{n+1}^{m} P_{C}\left(I-\mu_{m} B_{m}\right) y_{n+1}-\sum_{m=1}^{r} \eta_{n}^{m} P_{C}\left(I-\mu_{m} B_{m}\right) y_{n}\right\| \\
& =\| \sum_{m=1}^{r} \eta_{n+1}^{m} P_{C}\left(I-\mu_{m} B_{m}\right) y_{n+1}-\sum_{m=1}^{r} \eta_{n+1}^{m} P_{C}\left(I-\mu_{m} B_{m}\right) y_{n} \\
& \quad+\sum_{m=1}^{r} \eta_{n+1}^{m} P_{C}\left(I-\mu_{m} B_{m}\right) y_{n}-\sum_{m=1}^{r} \eta_{n}^{m} P_{C}\left(I-\mu_{m} B_{m}\right) y_{n} \| \\
& \leq\left\|y_{n+1}-y_{n}\right\|+M \sum_{m=1}^{r}\left|\eta_{n+1}^{m}-\eta_{n}^{m}\right|,
\end{aligned}
$$$$
\text { where } M=\max \left\{\sup \left\{\left\|P_{C}\left(I-\mu_{m} B_{m}\right) y_{n}\right\|: n \geq 1\right\}: 1 \leq m \leq\right.
$$
$r$, so

$$
\begin{aligned}
\| z_{n+1}- & z_{n}\|-\| x_{n+1}-x_{n} \| \\
\leq & \frac{\alpha_{n+1}}{1-\beta_{n+1}}\left(\left\|\gamma f\left(x_{n+1}\right)\right\|+\left\|A W_{n+1} \rho_{n+1}\right\|\right) \\
& +\frac{\alpha_{n}}{1-\beta_{n}}\left(\left\|\gamma f\left(x_{n}\right)\right\|+\left\|A W_{n} \rho_{n}\right\|\right) \\
& +\left\|y_{n+1}-y_{n}\right\|+M \sum_{m=1}^{r}\left|\eta_{n+1}^{m}-\eta_{n}^{m}\right| \\
& +\left\|W_{n+1} \rho_{n}-W_{n} \rho_{n}\right\|-\left\|x_{n+1}-x_{n}\right\| .
\end{aligned}
$$


Observing $y_{n}=V_{r_{n}} x_{n}, y_{n+1}=V_{r_{n+1}} x_{n+1}$, we have

$$
\begin{gathered}
\phi\left(y_{n}, \eta\right)+\frac{1}{r_{n}}\left\langle\eta-y_{n}, y_{n}-x_{n}\right\rangle \geq 0, \quad \forall \eta \in C, \\
\phi\left(y_{n+1}, \eta\right)+\frac{1}{r_{n+1}}\left\langle\eta-y_{n+1}, y_{n+1}-x_{n+1}\right\rangle \geq 0, \quad \forall \eta \in C .
\end{gathered}
$$

Putting $\eta=y_{n+1}$ in (19), $\eta=y_{n}$ in (20), adding up these two inequalities, and using condition $\left(A_{2}\right)$ to simplify, we have

$$
\begin{aligned}
& \left\langle y_{n+1}-y_{n}, y_{n}-y_{n+1}+y_{n+1}\right. \\
& \left.\quad-x_{n}-\frac{r_{n}}{r_{n+1}}\left(y_{n+1}-x_{n+1}\right)\right\rangle \geq 0 .
\end{aligned}
$$

By condition $\left(C_{4}\right)$, without loss of generality, we can assume that there exists a real number $m$ such that $r_{n}>m>0$, so

$$
\begin{aligned}
\left\|y_{n+1}-y_{n}\right\|^{2} \leq & \left\|y_{n+1}-y_{n}\right\| \\
& \times\left(\left\|x_{n+1}-x_{n}\right\|+\left|1-\frac{r_{n}}{r_{n+1}}\right|\left(y_{n+1}-x_{n+1}\right)\right) \\
\left\|y_{n+1}-y_{n}\right\| & \leq\left\|x_{n+1}-x_{n}\right\|+\frac{M_{1}}{m}\left|r_{n+1}-r_{n}\right|,
\end{aligned}
$$

where $M_{1}=\sup _{n \geq 1}\left\|y_{n}-x_{n}\right\|$.

Since $S_{i}, U_{n, i}$ are nonexpansive, so $\left\|W_{n+1} \rho_{n}-W_{n} \rho_{n}\right\| \leq$ $\prod_{i=1}^{n+1} \lambda_{i}\left\|S_{n+1} \rho_{n}-\rho_{n}\right\| \leq L\left(\prod_{i=1}^{n+1} \lambda_{i}\right)$, where $L=\sup _{n \geq 1} \| S_{n+1} \rho_{n}-$ $\rho_{n} \|$. Consider

$$
\begin{aligned}
\left\|z_{n+1}-z_{n}\right\|-\left\|x_{n+1}-x_{n}\right\| \\
\leq \frac{\alpha_{n+1}}{1-\beta_{n+1}}\left(\left\|\gamma f\left(x_{n+1}\right)\right\|+\left\|A W_{n+1} \rho_{n+1}\right\|\right) \\
\quad+\frac{\alpha_{n}}{1-\beta_{n}}\left(\left\|\gamma f\left(x_{n}\right)\right\|+\left\|A W_{n} \rho_{n}\right\|\right) \\
\quad+\frac{M_{1}}{m}\left|r_{n+1}-r_{n}\right|+L\left(\prod_{i=1}^{n+1} \lambda_{i}\right) .
\end{aligned}
$$

Using $0<\lambda_{i} \leq b<1(i \geq 1)$ and the conditions $\left(C_{1}\right)-\left(C_{3}\right)$, $\lim \sup _{n \rightarrow \infty}\left(\left\|z_{n+1}-z_{n}\right\|-\left\|x_{n+1}-x_{n}\right\|\right) \leq 0$. By Lemma 5, we conclude that $\lim _{n \rightarrow \infty}\left\|z_{n}-x_{n}\right\|=0, \lim _{n \rightarrow \infty}\left\|x_{n+1}-x_{n}\right\|=$ $\lim _{n \rightarrow \infty}\left(1-\beta_{n}\right)\left\|z_{n}-x_{n}\right\|=0$. Consider

$$
\begin{aligned}
\left\|\rho_{n+1}-\rho_{n}\right\| & \leq\left\|y_{n+1}-y_{n}\right\|+M \sum_{m=1}^{r}\left|\eta_{n+1}^{m}-\eta_{n}^{m}\right| \\
& \leq\left\|x_{n+1}-x_{n}\right\| \frac{M_{1}}{m}\left|r_{n+1}-r_{n}\right|+M \sum_{m=1}^{r}\left|\eta_{n+1}^{m}-\eta_{n}^{m}\right| .
\end{aligned}
$$

So $\lim _{n \rightarrow \infty}\left\|\rho_{n+1}-\rho_{n}\right\|=0$.
Step 3. Consider

$$
\begin{aligned}
\left\|x_{n}-W_{n} \rho_{n}\right\|= & \left\|x_{n}-x_{n+1}+x_{n+1}-W_{n} \rho_{n}\right\| \\
\leq & \left\|x_{n}-x_{n+1}\right\| \\
& +\| \alpha_{n} \gamma f\left(x_{n}\right)+\beta_{n} x_{n} \\
& \quad+\left(\left(1-\beta_{n}\right) I-\alpha_{n} A\right) W_{n} \rho_{n}-W_{n} \rho_{n} \| \\
\leq & \left\|x_{n+1}-x_{n}\right\|+\alpha_{n}\left\|\gamma f\left(x_{n}\right)-A W_{n} \rho_{n}\right\| \\
& +\beta_{n}\left\|x_{n}-W_{n} \rho_{n}\right\| \\
\left\|x_{n}-W_{n} \rho_{n}\right\| \leq & \frac{1}{1-\beta_{n}}\left\|x_{n+1}-x_{n}\right\| \\
& +\frac{\alpha_{n}}{1-\beta_{n}}\left\|\gamma f\left(x_{n}\right)-A W_{n} \rho_{n}\right\| .
\end{aligned}
$$

So we have $\lim _{n \rightarrow \infty}\left\|x_{n}-W_{n} \rho_{n}\right\|=0$.

Step 4. For any given $p \in \mathscr{F}$,

$$
\begin{aligned}
\left\|y_{n}-p\right\|^{2} & =\left\|V_{r_{n}} x_{n}-p\right\|^{2}=\left\langle V_{r_{n}} x_{n}-V_{r_{n}} p, x_{n}-p\right\rangle \\
& =\left\langle y_{n}-p, x_{n}-p\right\rangle \\
& =\frac{1}{2}\left(\left\|y_{n}-p\right\|^{2}+\left\|x_{n}-p\right\|^{2}-\left\|x_{n}-y_{n}\right\|^{2}\right),
\end{aligned}
$$

$\left\|x_{n+1}-p\right\|^{2}$

$$
=\| \alpha_{n} \gamma f\left(x_{n}\right)+\beta_{n} x_{n}
$$$$
+\left(\left(1-\beta_{n}\right) I-\alpha_{n} A\right) W_{n} \rho_{n}-p \|^{2}
$$$$
=\|\left[\left(I-\alpha_{n} A\right)\left(W_{n} \rho_{n}-p\right)+\beta_{n}\left(x_{n}-W_{n} \rho_{n}\right)\right]
$$$$
+\alpha_{n}\left(\gamma f\left(x_{n}\right)-A p\right) \|
$$$$
\leq\left\|\left(I-\alpha_{n} A\right)\left(W_{n} \rho_{n}-p\right)+\beta_{n}\left(x_{n}-W_{n} \rho_{n}\right)\right\|^{2}
$$$$
+2 \alpha_{n}\left\langle\gamma f\left(x_{n}\right)-A p, x_{n+1}-p\right\rangle
$$$$
\leq\left[\left(1-\alpha_{n} \bar{\gamma}\right)\left\|\rho_{n}-p\right\|+\beta_{n}\left\|x_{n}-W_{n} \rho_{n}\right\|\right]^{2}
$$$$
+2 \alpha_{n}\left\|\gamma f\left(x_{n}\right)-A p\right\| \cdot\left\|x_{n+1}-p\right\|
$$$$
=\left(1-\alpha_{n} \bar{\gamma}\right)^{2}\left\|\rho_{n}-p\right\|^{2}+\beta_{n}^{2}\left\|x_{n}-W_{n} \rho_{n}\right\|^{2}
$$$$
+2\left(1-\alpha_{n} \bar{\gamma}\right) \beta_{n}\left\|\rho_{n}-p\right\| \cdot\left\|x_{n}-W_{n} \rho_{n}\right\|
$$$$
+2 \alpha_{n}\left\|\gamma f\left(x_{n}\right)-A p\right\| \cdot\left\|x_{n+1}-p\right\|
$$$$
\leq\left(1-\alpha_{n} \bar{\gamma}\right)^{2}\left[\left\|x_{n}-p\right\|^{2}-\left\|x_{n}-y_{n}\right\|^{2}\right]
$$

$+\beta_{n}^{2}\left\|x_{n}-W_{n} \rho_{n}\right\|^{2}$

$+2\left(1-\alpha_{n} \bar{\gamma}\right) \beta_{n}\left\|\rho_{n}-p\right\| \cdot\left\|x_{n}-W_{n} \rho_{n}\right\|$

$+2 \alpha_{n}\left\|\gamma f\left(x_{n}\right)-A p\right\| \cdot\left\|x_{n+1}-p\right\|$. 
Simplifying it, we have

$$
\begin{aligned}
&\left(1-\alpha_{n} \bar{\gamma}\right)^{2}\left\|x_{n}-y_{n}\right\|^{2} \\
& \leq\left\|x_{n}-p\right\|^{2}-\left\|x_{n+1}-p\right\|^{2} \\
&+\alpha_{n}^{2} \bar{\gamma}^{2}\left\|x_{n}-p\right\|^{2}+\beta_{n}^{2}\left\|x_{n}-W_{n} \rho_{n}\right\|^{2} \\
&+2\left(1-\alpha_{n} \bar{\gamma}\right) \beta_{n}\left\|\rho_{n}-p\right\| \cdot\left\|x_{n}-W_{n} \rho_{n}\right\| \\
&+2 \alpha_{n}\left\|\gamma f\left(x_{n}\right)-A p\right\| \cdot\left\|x_{n+1}-p\right\| \\
& \leq\left(\left\|x_{n}-p\right\|+\left\|x_{n+1}-p\right\|\right)\left\|x_{n}-x_{n+1}\right\| \\
&+\alpha_{n}^{2} \bar{\gamma}^{2}\left\|x_{n}-p\right\|^{2}+\beta_{n}^{2}\left\|x_{n}-W_{n} \rho_{n}\right\|^{2} \\
&+2\left(1-\alpha_{n} \bar{\gamma}\right) \beta_{n}\left\|\rho_{n}-p\right\| \cdot\left\|x_{n}-W_{n} \rho_{n}\right\| \\
&+2 \alpha_{n}\left\|\gamma f\left(x_{n}\right)-A p\right\| \cdot\left\|x_{n+1}-p\right\| .
\end{aligned}
$$

So $\lim _{n \rightarrow \infty}\left\|x_{n}-y_{n}\right\|=0$.

\section{Step 5. Consider}

$$
\begin{aligned}
& \left\|\rho_{n}-p\right\|^{2} \\
& =\left\|\sum_{m=1}^{r} \eta_{n}^{m} P_{C}\left(I-\mu_{m} B_{m}\right) y_{n}-p\right\|^{2} \\
& \leq\left\|\left(y_{n}-p\right)-\mu_{m}\left(B_{m} y_{n}-B_{m} p\right)\right\|^{2} \\
& =\left\|y_{n}-p\right\|^{2}+\mu_{m}^{2}\left\|B_{m} y_{n}-B_{m} p\right\|^{2} \\
& -2 \mu_{m}\left\langle y_{n}-p, B_{m} y_{n}-B_{m} p\right\rangle \\
& \leq\left\|y_{n}-p\right\|^{2}+\mu_{m}^{2}\left\|B_{m} y_{n}-B_{m} p\right\|^{2} \\
& -2 \mu_{m} l_{m}\left\|B_{m} y_{n}-B_{m} p\right\|^{2} \\
& \leq\left\|x_{n}-p\right\|^{2}+\mu_{m}\left(\mu_{m}-2 l_{m}\right)\left\|B_{m} y_{n}-B_{m} p\right\|^{2} \\
& \left\|x_{n+1}-p\right\|^{2} \\
& \leq\left(1-\alpha_{n} \bar{\gamma}\right)^{2}\left\|x_{n}-p\right\|^{2} \\
& +\left(1-\alpha_{n} \bar{\gamma}\right)^{2} \mu_{m}\left(\mu_{m}-2 l_{m}\right)\left\|B_{m} y_{n}-B_{m} p\right\|^{2} \\
& +2\left(1-\alpha_{n} \bar{\gamma}\right) \beta_{n}\left\|\rho_{n}-p\right\| \cdot\left\|x_{n}-W_{n} \rho_{n}\right\| \\
& +2 \alpha_{n}\left\|\gamma f\left(x_{n}\right)-A p\right\| \cdot\left\|x_{n+1}-p\right\| \\
& \left(2 l_{m}-\mu_{m}\right)\left(1-\alpha_{n} \bar{\gamma}\right)^{2} \mu_{m}\left\|B_{m} y_{n}-B_{m} p\right\|^{2} \\
& \leq\left(\left\|x_{n}-p\right\|+\left\|x_{n+1}-p\right\|\right)\left\|x_{n}-x_{n+1}\right\| \\
& +\alpha_{n}^{2} \bar{\gamma}^{2}\left\|x_{n}-p\right\|^{2} \\
& +2\left(1-\alpha_{n} \bar{\gamma}\right) \beta_{n}\left\|\rho_{n}-p\right\| \cdot\left\|x_{n}-W_{n} \rho_{n}\right\| \\
& +2 \alpha_{n}\left\|\gamma f\left(x_{n}\right)-A p\right\| \cdot\left\|x_{n+1}-p\right\| \text {. }
\end{aligned}
$$

So $\lim _{n \rightarrow \infty}\left\|B_{m} y_{n}-B_{m} p\right\|=0$.
Step 6. Consider

$$
\begin{aligned}
\left\|\rho_{n}-p\right\|^{2}=\left\|\sum_{m=1}^{r} \eta_{n}^{m} P_{C}\left(I-\mu_{m} B_{m}\right) y_{n}-p\right\|^{2} \\
\leq\left\langle\left(y_{n}-\mu_{m} B_{m} y_{n}\right)-\left(p-\mu_{m} B_{m} p\right), \rho_{n}-p\right\rangle \\
=\frac{1}{2}\left\{\left\|I-\mu_{m} B_{m} y_{n}-I-\mu_{m} B_{m} p\right\|^{2}+\left\|\rho_{n}-p\right\|^{2}\right. \\
\left.\quad-\left\|\left(y_{n}-\rho_{n}\right)-\mu_{m}\left(B_{m} y_{n}-B_{m} p\right)\right\|^{2}\right\} \\
\leq \frac{1}{2}\left\{\left\|y_{n}-p\right\|^{2}+\left\|\rho_{n}-p\right\|^{2}-\left\|y_{n}-\rho_{n}\right\|^{2}\right. \\
\quad-\mu_{m}^{2}\left\|B_{m} y_{n}-B_{m} p\right\|^{2} \\
\left.\quad+2 \mu_{m}\left\langle y_{n}-\rho_{n}, B_{m} y_{n}-B_{m} p\right\rangle\right\}
\end{aligned}
$$

$\left\|\rho_{n}-p\right\|^{2} \leq\left\|y_{n}-p\right\|^{2}-\left\|y_{n}-\rho_{n}\right\|^{2}-\mu_{m}^{2}\left\|B_{m} y_{n}-B_{m} p\right\|^{2}$

$$
+2 \mu_{m}\left\langle y_{n}-\rho_{n}, B_{m} y_{n}-B_{m} p\right\rangle
$$$$
\left\|x_{n+1}-p\right\|^{2}
$$

$$
\begin{aligned}
& \leq\left(1-\alpha_{n} \bar{\gamma}\right)^{2}\left\|\rho_{n}-p\right\|^{2}+\beta_{n}^{2}\left\|x_{n}-W_{n} \rho_{n}\right\|^{2} \\
&+2\left(1-\alpha_{n} \bar{\gamma}\right) \beta_{n}\left\|\rho_{n}-p\right\| \cdot\left\|x_{n}-W_{n} \rho_{n}\right\| \\
&+2 \alpha_{n}\left\|\gamma f\left(x_{n}\right)-A p\right\| \cdot\left\|x_{n+1}-p\right\| \\
& \leq\left(1-\alpha_{n} \bar{\gamma}\right)^{2}\left[\left\|y_{n}-p\right\|^{2}-\left\|y_{n}-\rho_{n}\right\|^{2}\right. \\
& \quad-\mu_{m}^{2}\left\|B_{m} y_{n}-B_{m} p\right\|^{2} \\
&\left.+2 \mu_{m}\left\langle y_{n}-\rho_{n}, B_{m} y_{n}-B_{m} p\right\rangle\right] \\
&+\beta_{n}^{2}\left\|x_{n}-W_{n} \rho_{n}\right\|^{2} \\
&+2\left(1-\alpha_{n} \bar{\gamma}\right) \beta_{n}\left\|\rho_{n}-p\right\| \cdot\left\|x_{n}-W_{n} \rho_{n}\right\| \\
&+ 2 \alpha_{n}\left\|\gamma f\left(x_{n}\right)-A p\right\| \cdot\left\|x_{n+1}-p\right\| \\
& \leq\left\|x_{n}-p\right\|^{2}+\alpha_{n} \bar{\gamma}^{2}\left\|x_{n}-p\right\|^{2} \\
&+ 2 \mu_{m}\left(1-\alpha_{n} \bar{\gamma}\right)^{2}\left\|y_{n}-\rho_{n}\right\| \cdot\left\|B_{m} y_{n}-B_{m} p\right\| \\
&-\left(1-\alpha_{n} \bar{\gamma}\right)^{2}\left\|y_{n}-\rho_{n}\right\|^{2}+\beta_{n}^{2}\left\|x_{n}-W_{n} \rho_{n}\right\|^{2} \\
&+2\left(1-\alpha_{n} \bar{\gamma}\right) \beta_{n}\left\|\rho_{n}-p\right\| \cdot\left\|x_{n}-W_{n} \rho_{n}\right\| \\
&+ 2 \alpha_{n}\left\|\gamma f\left(x_{n}\right)-A p\right\| \cdot\left\|x_{n+1}-p\right\| \\
&\left(1-\alpha_{n} \bar{\gamma}\right)^{2}\left\|y_{n}-\rho_{n}\right\|^{2} \\
& \leq\left(\left\|x_{n}-p\right\|+\left\|x_{n+1}-p\right\|\right)\left\|x_{n}-x_{n+1}\right\| \\
&+2 \mu_{m}\left(1-\alpha_{n} \bar{\gamma}\right)^{2}\left\|y_{n}-\rho_{n}\right\| \cdot\left\|B_{m} y_{n}-B_{m} p\right\| \\
&+\beta_{n}^{2}\left\|x_{n}-W_{n} \rho_{n}\right\|^{2}
\end{aligned}
$$




$$
\begin{aligned}
& +2\left(1-\alpha_{n} \bar{\gamma}\right) \beta_{n}\left\|\rho_{n}-p\right\| \cdot\left\|x_{n}-W_{n} \rho_{n}\right\| \\
& +2 \alpha_{n}\left\|\gamma f\left(x_{n}\right)-A p\right\| \cdot\left\|x_{n+1}-p\right\| .
\end{aligned}
$$

So $\lim _{n \rightarrow \infty}\left\|y_{n}-\rho_{n}\right\|=0$.

Step 7. Consider

$$
\begin{aligned}
\left\|y_{n}-W_{n} y_{n}\right\| \leq & \left\|W_{n} y_{n}-W_{n} \rho_{n}\right\| \\
& +\left\|W_{n} \rho_{n}-x_{n}\right\|+\left\|x_{n}-y_{n}\right\| \\
\leq & \left\|y_{n}-\rho_{n}\right\|+\left\|W_{n} \rho_{n}-x_{n}\right\|+\left\|x_{n}-y_{n}\right\| .
\end{aligned}
$$

So $\lim _{n \rightarrow \infty}\left\|y_{n}-W_{n} y_{n}\right\|=0$.

Step 8. Next, we prove that $\lim _{\sup _{n \rightarrow \infty}}\left\langle\gamma f(q)-A q, x_{n}-q\right\rangle \leq$ 0 , where $q=P_{\mathscr{F}}(\gamma f+(I-A q))$. that

To show it, we can choose a subsequence $\left\{x_{n_{i}}\right\}$ of $\left\{x_{n}\right\}$ such

$$
\begin{aligned}
\limsup _{n \rightarrow \infty} & \left\langle\gamma f(q)-A q, x_{n}-q\right\rangle \\
= & \lim _{i \rightarrow \infty}\left\langle\gamma f(q)-A q, x_{n_{i}}-q\right\rangle .
\end{aligned}
$$

Since $\left\{y_{n_{i}}\right\}$ is bounded, so there exists a subsequence $\left\{y_{n_{i j}}\right\}$ of $\left\{y_{n_{i}}\right\}$ which converges weakly to $\omega$. Without loss of generality, we can assume that $y_{n_{i}} \rightarrow \omega$; then, $\phi\left(y_{n}, \eta\right)+\left(1 / r_{n}\right)\left\langle\eta-y_{n}, y_{n}-\right.$ $\left.x_{n}\right\rangle \geq 0$, for all $\eta \in C,\left\langle\eta-y_{n},\left(y_{n}-x_{n}\right) / r_{n}\right\rangle \geq \phi\left(\eta, y_{n}\right)$, $\left\langle\eta-y_{n_{i}},\left(y_{n_{i}}-x_{n_{i}}\right) / r_{n_{i}}\right\rangle \geq \phi\left(\eta, y_{n_{i}}\right)$. So $\phi(\eta, \omega) \leq 0$.

For any $t$ with $0 \leq t \leq 1$ and $\eta \in C$, let $\eta_{t}=t \eta+(1-t) \omega$. Since $\eta \in C$ and $\phi\left(\eta_{t}, \omega\right) \leq 0$, from conditions $\left(A_{1}\right)$ and $\left(A_{4}\right)$, we have

$$
0=\phi\left(\eta_{t}, \eta_{t}\right) \leq t \phi\left(\eta_{t}, \eta\right)+(1-t) \phi\left(\eta_{t}, \omega\right) \leq t \phi\left(\eta_{t}, \eta\right) .
$$

This implies that $\phi\left(\eta_{t}, \eta\right) \geq 0$. From condition $\left(A_{3}\right)$, we have $\phi(\omega, \eta) \geq 0$. So $\omega \in \operatorname{EP}(\phi)$.

Define a mapping $J: C \rightarrow C$ by $J x=\sum_{m=1}^{r} \eta_{n}^{m} P_{C}(I-$ $\left.\mu_{m} B_{m}\right) x$, for all $x \in C$, where $\lim _{n \rightarrow \infty} \eta_{n}^{m}=\eta^{m}$. From Lemma 7 we see that $J$ is nonexpansive such that

$$
\begin{gathered}
F(J)=\bigcap_{m=1}^{r} F\left(P_{C}\left(I-\mu_{m} B_{m}\right)\right)=\bigcap_{m=1}^{r} \operatorname{VI}\left(C, B_{m}\right) . \\
\left\|y_{n}-J y_{n}\right\| \leq\left\|y_{n}-\rho_{n}\right\|+\left\|\rho_{n}-J y_{n}\right\| \\
=\left\|y_{n}-\rho_{n}\right\|+\| \sum_{m=1}^{r} \eta_{n}^{m} P_{C}\left(I-\mu_{m} B_{m}\right) y_{n} \\
\quad-\sum_{m=1}^{r} \eta^{m} P_{C}\left(I-\mu_{m} B_{m}\right) y_{n} \| \\
\leq\left\|y_{n}-\rho_{n}\right\|+M \sum_{m=1}^{r}\left|\eta_{n}^{m}-\eta^{m}\right| \longrightarrow 0
\end{gathered}
$$

Since every nonexpansive mapping is strictly pseudocontractive, so $\omega \in F(J)=\bigcap_{m=1}^{r} \operatorname{VI}\left(C, B_{m}\right)$.

Now we prove that $\omega \in F(\omega)$, and if not, we have $\omega \neq W(\omega)$. From Opial's condition, we have

$$
\begin{aligned}
\liminf _{i \rightarrow \infty}\left\|y_{n_{i}}-\omega\right\| & <\liminf _{i \rightarrow \infty}\left\|y_{n_{i}}-W \omega\right\| \\
\leq & \liminf _{i \rightarrow \infty}\left(\left\|y_{n_{i}}-W y_{n_{i}}\right\|+\left\|W y_{n_{i}}-W \omega\right\|\right) \\
\leq & \liminf _{i \rightarrow \infty}\left(\left\|y_{n_{i}}-W y_{n_{i}}\right\|+\left\|y_{n_{i}}-\omega\right\|\right), \\
\lim _{i \rightarrow \infty}\left\|W y_{n_{i}}-y_{n_{i}}\right\| \leq & \lim _{i \rightarrow \infty}\left(\left\|W y_{n_{i}}-W_{n_{i}} y_{n_{i}}\right\|+\left\|W_{n_{i}} y_{n_{i}}-y_{n_{i}}\right\|\right) \\
\leq & \lim _{i \rightarrow \infty}\left\{\sup _{x \in C}\left\|W \omega-W_{n_{i}} \omega\right\|\right\} \\
& +\lim _{i \rightarrow \infty}\left\|W_{n_{i}} y_{n_{i}}-y_{n_{i}}\right\|=0 .
\end{aligned}
$$

Therefore, $\liminf \inf _{i \rightarrow \infty}\left\|y_{n_{i}}-\omega\right\|<\liminf _{i \rightarrow \infty}\left\|y_{n_{i}}-\omega\right\|$, so $\omega \in F(\omega)$.

Step 9. Finally, we prove that $x_{n} \rightarrow q=P_{\mathscr{F}}(\gamma f+(I-A) q)$. Since $q=P_{\mathscr{F}}(\gamma f+(I-A) q)$, so

$$
\begin{aligned}
\limsup _{n \rightarrow \infty} & \left\langle\gamma f(q)-A q, x_{n}-q\right\rangle \\
& =\lim _{i \rightarrow \infty}\left\langle\gamma f(q)-A q, x_{n_{i}}-q\right\rangle \\
& =\lim _{i \rightarrow \infty}\left\langle\gamma f(q)-A q, x_{n_{i}}-y_{n_{i}}+y_{n_{i}}-q\right\rangle \\
& =\langle\gamma f(q)-A q, \omega-q\rangle \leq 0,
\end{aligned}
$$

$\left\|x_{n+1}-q\right\|^{2}$

$$
\begin{aligned}
= & \| \alpha_{n}\left(\gamma f\left(x_{n}\right)-A q\right) \\
& +\left[\left(1-\beta_{n} I-\alpha_{n} A\right)\left(W_{n} \rho_{n}-q\right)+\beta_{n}\left(x_{n}-q\right)\right] \|^{2} \\
\leq & \left.\|\left(1-\beta_{n} I-\alpha_{n} A\right)\left(W_{n} \rho_{n}-q\right)+\beta_{n}\left(x_{n}-q\right)\right] \|^{2} \\
& +2 \alpha_{n} \gamma\left\langle f\left(x_{n}\right)-f(q), x_{n+1}-q\right\rangle \\
& +2 \alpha_{n}\left\langle\gamma f(q)-A q, x_{n+1}-q\right\rangle \\
\leq & {\left[\left(1-\beta_{n}-\alpha_{n} \bar{\gamma}\right)\left\|\rho_{n}-p\right\|+\beta_{n}\left\|x_{n}-q\right\|\right]^{2} } \\
& +2 \alpha_{n} \gamma h\left\|x_{n}-q\right\| \cdot\left\|x_{n+1}-q\right\| \\
& +2 \alpha_{n}\left\langle\gamma f(q)-A q, x_{n+1}-q\right\rangle \\
\leq & \left(1-\alpha_{n} \bar{\gamma}\right)^{2}\left\|x_{n}-q\right\|^{2} \\
& +\alpha_{n} \gamma h\left(\left\|x_{n}-q\right\|^{2}+\left\|x_{n+1}-q\right\|^{2}\right) \\
& +2 \alpha_{n}\left\langle\gamma f(q)-A q, x_{n+1}-q\right\rangle .
\end{aligned}
$$


This implies that

$$
\begin{aligned}
& \left\|x_{n+1}-q\right\|^{2} \\
& \leq\left[1-\frac{2(\bar{\gamma}-\gamma h) \alpha_{n}}{1-\alpha_{n} \gamma h}\right]\left\|x_{n}-q\right\|^{2} \\
& \quad+\frac{2(\bar{\gamma}-\gamma h) \alpha_{n}}{1-\alpha_{n} \gamma h}\left\{\frac{\alpha_{n} \gamma^{2}}{2(\bar{\gamma}-\gamma h)}\left\|x_{n}-q\right\|^{2}\right. \\
& \left.+\frac{1}{\bar{\gamma}-\gamma h}\left\langle\gamma f(q)-A q, x_{n+1}-q\right\rangle\right\} .
\end{aligned}
$$

From Lemma $6,\left\{x_{n}\right\}$ converges strongly to $q$.

Taking $F(x, y)=0, D=0$ for all $x, y \in H, r_{n}=1, m=1$ in Theorem 10, then Theorem 10 is reduced to the following.

Corollary 11. Let $C$ be a nonempty closed convex subset of a real Hilbert space $H$. Let $B: C \rightarrow H$ be a $\lambda$-inverse strongly monotone mapping and $D: C \rightarrow H$ a $\alpha$-inverse strongly monotone mapping. Let $\left\{T_{n}\right\}_{n=1}^{\infty}: C \rightarrow C$ be a family of infinite $k_{n}$-strict pseudocontractive mappings with $0 \leq k_{n}<1$ and let $\left\{\lambda_{n}\right\}_{n=1}^{\infty}$ be a sequence of positive numbers in $[0, b]$ for some $b \in(0,1) .\left\{S_{n}\right\}_{n=1}^{\infty}: C \rightarrow C$ is a family of infinitely nonexpansive mappings such that $\mathscr{F}=F(W) \bigcap \operatorname{VI}(C, B) \neq \emptyset$, where $F(W):=\bigcap_{n=1}^{\infty} F\left(S_{n}\right)$. Let $A$ be a strongly positive linear bounded operator with coefficient $\bar{\gamma}>0$ and let $f: H \rightarrow H$ be a contraction with contraction constant $h(0<h<1)$ and $0<\gamma<(\bar{\gamma} / h)$. Let $\left\{x_{n}\right\}$ and $\left\{y_{n}\right\}$ be generated in sequence by $x_{1} \in H$ and

$$
\begin{gathered}
y_{n}=P_{C}(I-\lambda B) x_{n}, \\
x_{n+1}=\alpha_{n} \gamma f\left(x_{n}\right)+\beta_{n} x_{n}+\left(\left(1-\beta_{n}\right) I-\alpha_{n} A\right) W_{n} y_{n}
\end{gathered}
$$

where $\lambda \in(0,2 \alpha],\left\{\alpha_{n}\right\},\left\{\beta_{n}\right\} \subset[0,1]$. If the following conditions are satisfied:

$$
\begin{aligned}
& \left(C_{1}\right) \lim _{n \rightarrow \infty} \alpha_{n}=0, \sum_{n=1}^{\infty} \alpha_{n}=\infty ; \\
& \left(C_{2}\right) 0<\liminf _{n \rightarrow \infty} \beta_{n} \leq \lim \sup _{n \rightarrow \infty} \beta_{n}<1,
\end{aligned}
$$

then $\left\{x_{n}\right\}$ converges strongly to $q \in \mathscr{F}$, where $q=P_{\mathscr{F}}(\gamma f+(I-$ A)) $q$.

\section{Conflict of Interests}

The authors declare that there is no conflict of interests regarding the publication of this paper.

\section{Acknowledgments}

The authors acknowledge the support of the Foundation of Langfang Teachers College (LSZY201307) and the Foundation of Education Department of Hebei Province (2011169).

\section{References}

[1] W. Takahashi and M. Toyoda, "Weak convergence theorems for nonexpansive mappings and monotone mappings," Journal of
Optimization Theory and Applications, vol. 118, no. 2, pp. 417428, 2003.

[2] Y. Yao and J. C. Yao, "On modified iterative method for nonexpansive mappings and monotone mappings," Applied Mathematics and Computation, vol. 186, no. 2, pp. 1551-1558, 2007.

[3] S. S. Chang, H. W. Joseph Lee, and C. K. Chan, "A new method for solving equilibrium problem fixed point problem and variational inequality problem with application to optimization," Nonlinear Analysis, Theory, Methods \& Applications, vol. 70, no. 9, pp. 3307-3319, 2009.

[4] L. Min and S. S. Chang, "A new iterative method for finding common solutions of generalized equilibrium problem, fixed point problem of infinite k-strict pseudo-contractive mappings, and quasi-variational inclusion problem," Acta Mathematica Scientia, vol. 32, no. 2, pp. 499-519, 2012.

[5] S. Plubtieng and R. Punpaeng, "A general iterative method for equilibrium problems and fixed point problems in Hilbert spaces," Journal of Mathematical Analysis and Applications, vol. 336, no. 1, pp. 455-469, 2007.

[6] S. Takahashi and W. Takahashi, "Viscosity approximation methods for equilibrium problems and fixed point problems in Hilbert spaces," Journal of Mathematical Analysis and Applications, vol. 331, no. 1, pp. 506-515, 2007.

[7] A. Tada and W. Takahashi, "Strong convergence theorem for an equilibrium problem and a nonexpansive mapping," Journal of Optimization Theory and Applications, vol. 133, pp. 359-370, 2007.

[8] C. Gang and H. Changsong, "A hybrid approximation method for equilibrium and fixed point problems for a family of infinitely nonexpansive mappings and a monotone mapping," Nonlinear Analysis: Hybrid Systems, vol. 3, no. 4, pp. 395-407, 2009.

[9] L. Ying, "Weak convergence theorem for equilibrium and varitional inequality problems and a in nitely nonexpansive mappings in Hilbert space," Mathematica Applicata, vol. 26, pp. 292-299, 2013.

[10] S. Yong-Cho and S.-M. Kang, "Approximation of common solutions of varitional inequalities via strict pseudocontractions," Acta Mathematica Scientia, vol. 32, no. 4, pp. 1607-1618, 2012.

[11] K. Shimoji and W. Takahashi, "Strong convergence to common fixed points of infinite nonexpansive mappings and applications," Taiwanese Journal of Mathematics, vol. 5, no. 2, pp. 387404, 2001. 


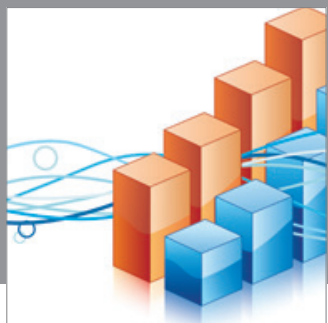

Advances in

Operations Research

mansans

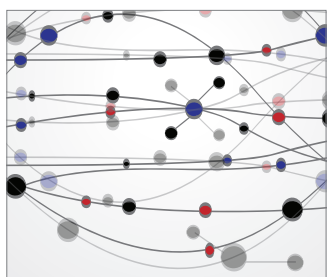

The Scientific World Journal
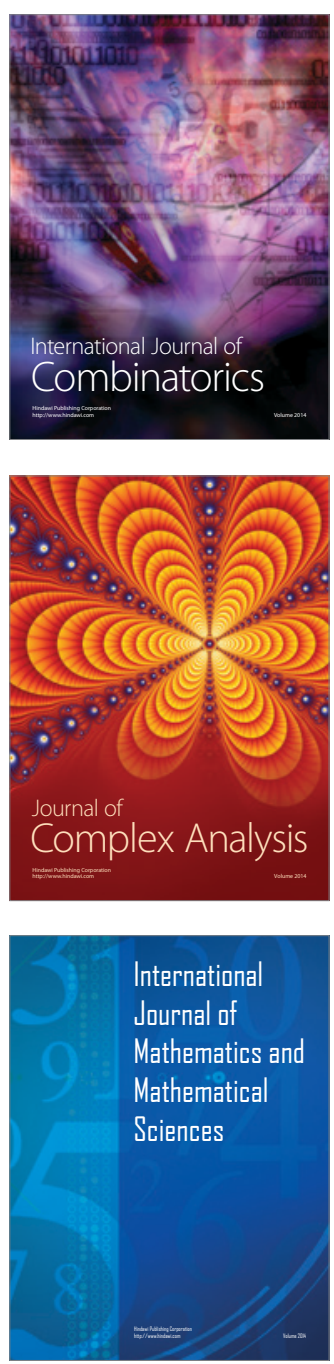
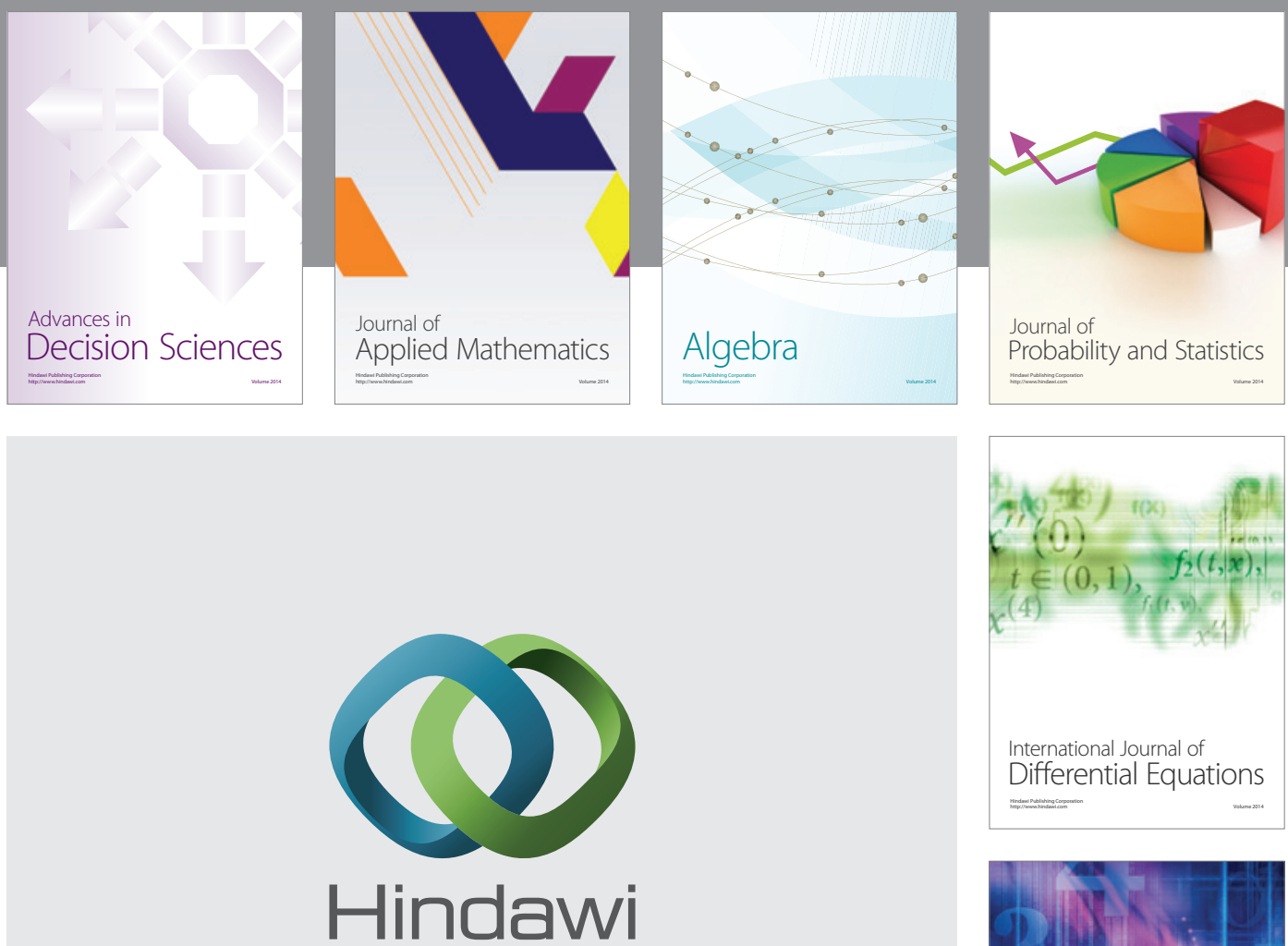

Submit your manuscripts at http://www.hindawi.com
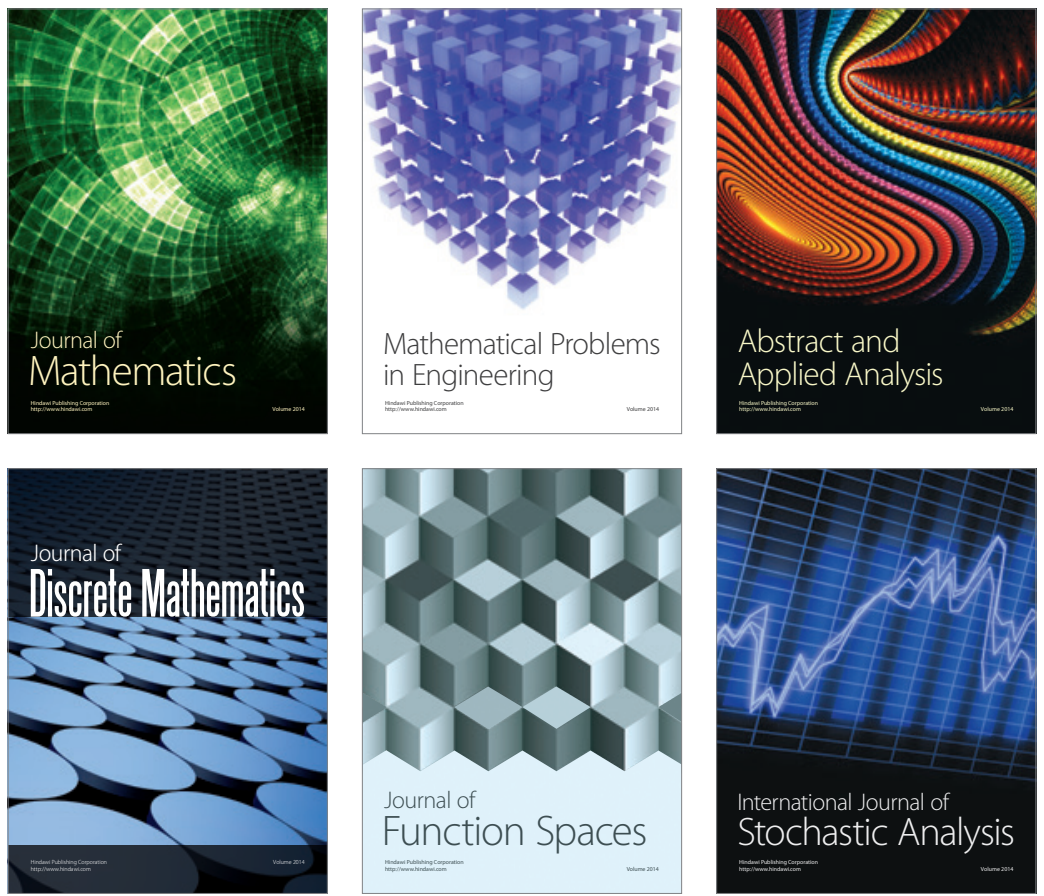

Journal of

Function Spaces

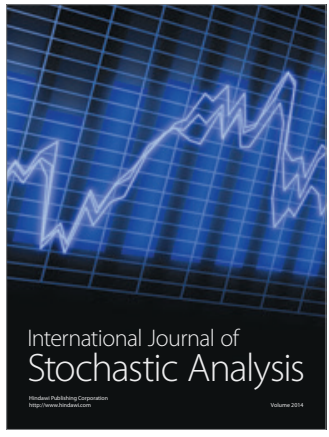

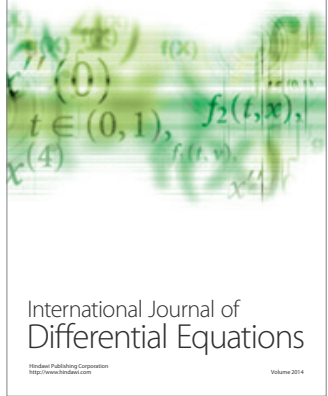
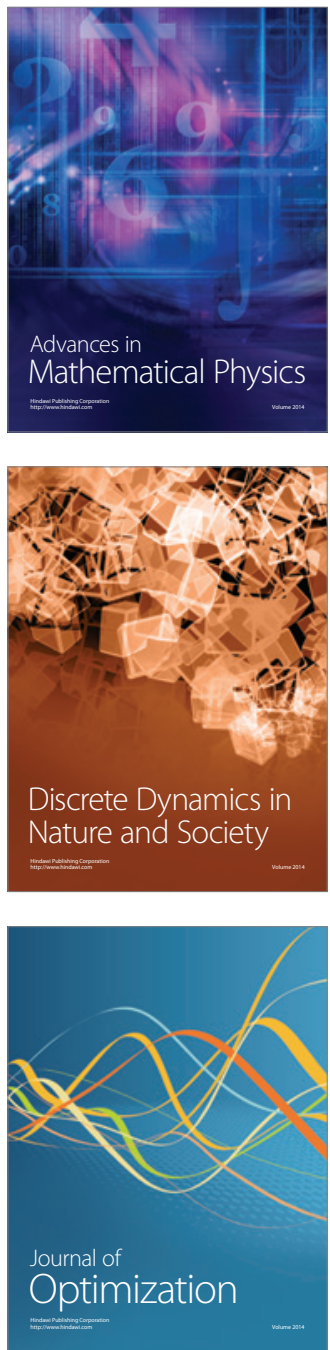